\title{
Spectral Functions from Hadronic $\tau$ Decays and QCD
}

\author{
Michel Davier \\ Laboratoire de l'Accélérateur Linéaire \\ IN2P3/CNRS et Université de Paris-Sud \\ 91898 Orsay, France \\ E-mail: davier@lal.in2p3.fr
}

\begin{abstract}
Hadronic decays of the $\tau$ lepton provide a clean environment to study hadron dynamics in an energy regime dominated by resonances, with the interesting information captured in the spectral functions. Recent results from ALEPH on exclusive channels are presented, with some emphasis on the $\pi \pi^{0}$ final state which plays a crucial role for the determination of the hadronic contribution to the muon anomalous magnetic moment. Inclusive spectral functions are the basis for QCD analyses, delivering an accurate determination of the strong coupling constant, quantitative information on nonperturbative contributions and a measurement of the mass of the strange quark.
\end{abstract}

\section{Introduction}

Hadrons produced in $\tau$ decays are born out of the charged weak current, i.e. out of the QCD vacuum. This property guarantees that hadronic physics factorizes in these processes which are then completely characterized for each decay channel by spectral functions as far as the total rate is concerned. Furthermore, the produced hadronic systems have $I=1$ and spin-parity $J^{P}=0^{+}, 1^{-}(\mathrm{V})$ and $J^{P}=0^{-}, 1^{+}(\mathrm{A})$. The spectral functions are directly related to the invariant mass spectra of the hadronic final states, normalized to their respective branching ratios and corrected for the $\tau$ decay kinematics. For a given spin-1 vector decay, one has

$$
\begin{gathered}
v(s) \equiv \frac{m_{\tau}^{2}}{6\left|V_{u d}\right|^{2} S_{\mathrm{EW}}} \frac{B\left(\tau^{-} \rightarrow V^{-} \nu_{\tau}\right)}{B\left(\tau^{-} \rightarrow e^{-} \bar{\nu}_{e} \nu_{\tau}\right)} \\
\times \frac{d N_{V}}{N_{V} d s}\left[\left(1-\frac{s}{m_{\tau}^{2}}\right)^{2}\left(1+\frac{2 s}{M_{\tau}^{2}}\right)\right]^{-1}
\end{gathered}
$$

where $V_{u d}=0.9748 \pm 0.0010$ denotes the CKM weak mixing matrix element [1] and $S_{\mathrm{EW}}$ accounts for electroweak radiative corrections (see below). Isospin symmetry (CVC) connects the $\tau$ and $e^{+} e^{-}$annihilation spectral functions, the latter being proportional to the $\mathrm{R}$ ratio.

Hadronic $\tau$ decays are a clean probe of hadron dynamics in an interesting energy region dom- inated by resonances. However, perturbative QCD can be seriously considered due to the relatively large $\tau$ mass. Many hadronic modes have been measured and studied, while some earlier discrepancies (before 1990) have been resolved with high-statistics and low-systematics experiments. Samples of $\sim 4 \times 10^{5}$ measured decays are available in each LEP experiment and CLEO. Conditions for low systematic uncertainties are particularly well met at LEP: measured samples have small non- $\tau$ backgrounds $(\sim 1 \%)$ and large selection efficiency (92\%), for example in ALEPH.

Recent results in the field are discussed in this report.

\section{New ALEPH Spectral Functions}

Preliminary spectral functions based on the full LEP1 statistics are available from ALEPH. The corresponding results for the branching fractions are given separately [2]. The analysis uses an improved treatment of photons as compared to the published analyses based on a reduced sample $[3,4]$. Spectral functions are unfolded from the measured mass spectra after background subtraction using a mass-migration matrix obtained from the simulation in order to account for detector and reconstruction biases. Backgrounds from non- $\tau \tau$ events are small $(<1 \%$ and subtractions are dominated by $\tau$ decay feedthroughs. This is 

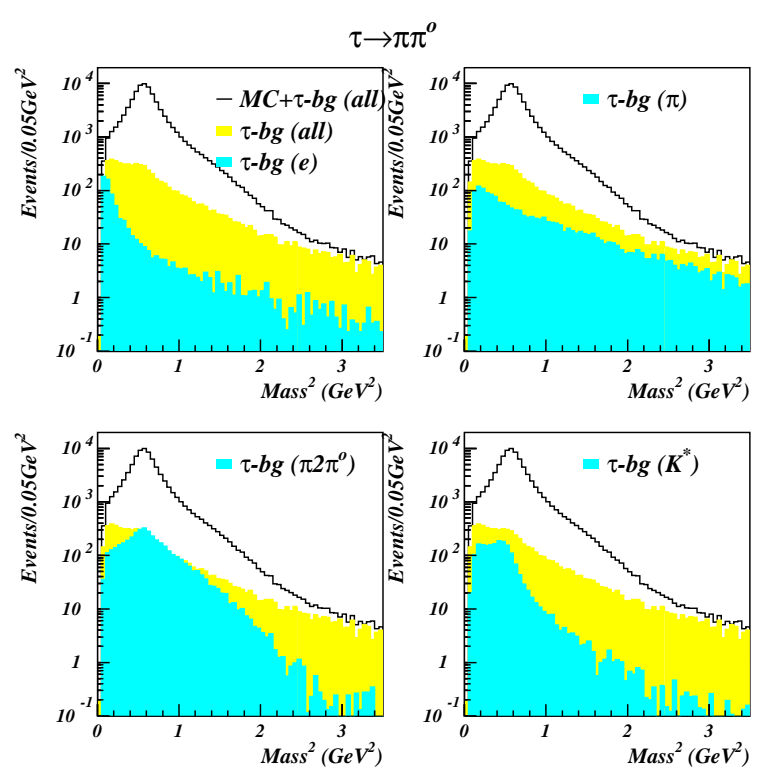

Figure 1. Feedthrough in the $\pi \pi^{0}$ channel from other $\tau$ decay modes in the ALEPH analysis, as estimated from the Monte Carlo simulation. The light-shaded histogram indicates the total feedthrough, while the dark-shaded histograms in each plot show the dominant individual sources.

illustrated in Fig. 1 for the $\pi \pi^{0}$ channel, where the main feedthrough contributions are from the $\pi$ mode at large masses (extra photon from radiation and fake photons from hadronic interactions in the electromagnetic calorimeter) and mostly the $a_{1} \rightarrow \pi 2 \pi^{0}$ mode (one $\pi^{0}$ lost).

The raw and unfolded spectra for the $\pi^{-} \pi^{0}$ mode are given in Fig. 2. Apart from the normalization effects studied in the branching ratio analysis [2], systematic biases affecting the mass spectra, are studied separately. The corresponding uncertainties and correlations in mass are incorporated in covariance matrices for every considered channel. According to the number of pions in the final state, the spectral functions are separated into vector and axial-vector components. As for final states involving $K \bar{K}$ pairs, specific input is required to achieve the $V-A$ separation [5]. Strange final states are treated separately.

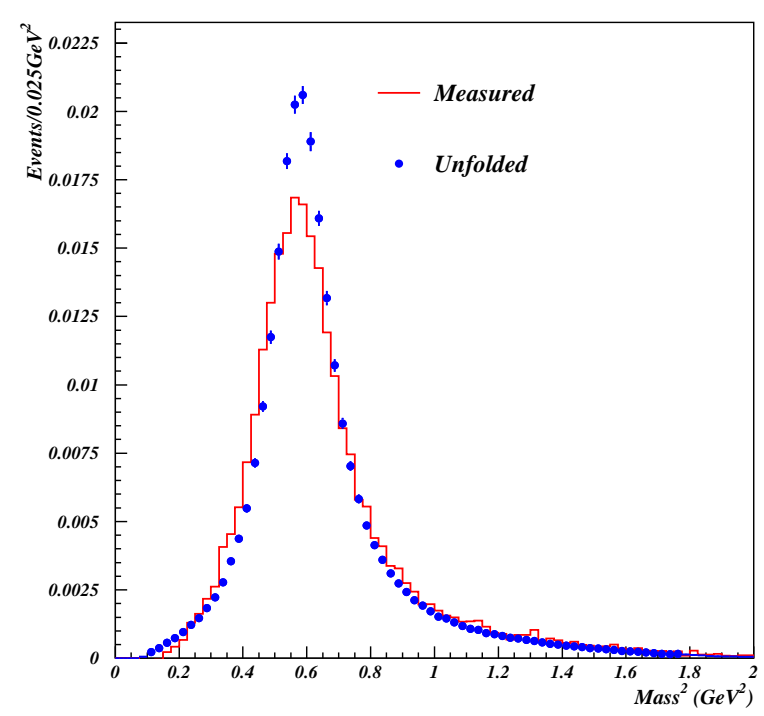

Figure 2. Spectral functions in the $\pi \pi^{0}$ mode from ALEPH before (histogram) and after (points) the unfolding procedure.

\section{Specific Final States}

\subsection{The $2 \pi$ Vector State}

\subsubsection{The Data}

The spectral function from $\tau \rightarrow \nu_{\tau} \pi^{-} \pi^{0}$ in the full-LEP1 ALEPH analysis $\left(\sim 10^{5}\right.$ events $)$ is presented in Fig 3. It is dominated by the $\rho(770)$ resonance, with some broad structure around $1.25 \mathrm{GeV}$.

Results from CLEO [6] are in good agreement with the ALEPH data. The statistics is comparable in the two cases, however whereas the acceptance is very flat across the mass spectrum in the case of ALEPH, a strong increase is observed for the CLEO case. As a consequence, ALEPH data are more precise below the $\rho$ peak, while CLEO is more precise above. Thus the two pieces of data are complementary. The comparison is given in Fig. 4.

\subsection{2. $\pi \pi$ Spectral Functions and $\pi$ Form Factors}

It is useful to carefully write down all the factors involved in the comparison of $e^{+} e^{-}$and $\tau$ 


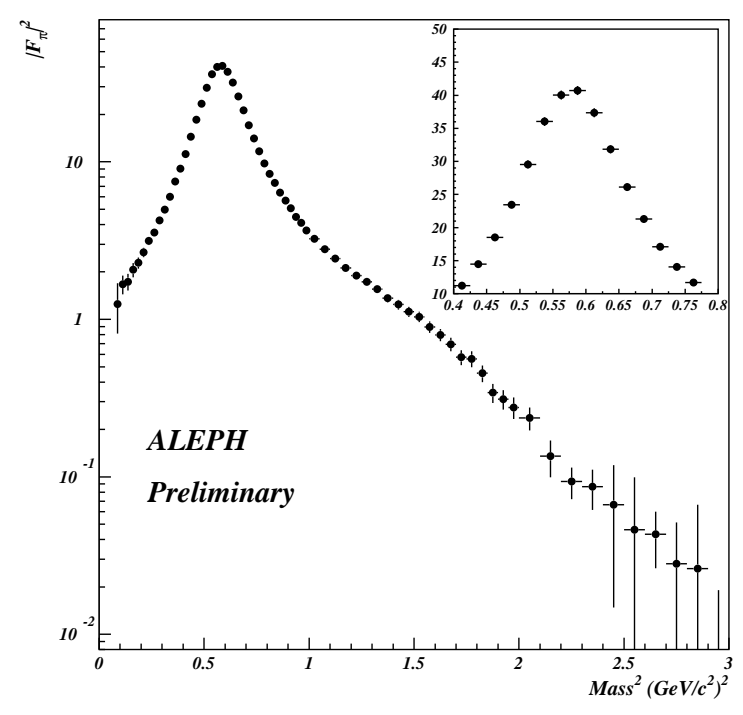

Figure 3. The pion form factor squared $\left|F_{\pi}^{-}(s)\right|^{2}$ measured by ALEPH. Because of the unfolding procedure, the errors in nearby bins are strongly correlated (only diagonal errors are shown).

spectral functions in order to make explicit the possible sources of CVC breaking. On the $e^{+} e^{-}$ side we have

$$
\begin{aligned}
\sigma\left(e^{+} e^{-} \longrightarrow \pi^{+} \pi^{-}\right) & =\frac{4 \pi \alpha^{2}}{s} v_{0}(s) \\
v_{0}(s) & =\frac{\beta_{0}^{3}(s)}{12 \pi}\left|F_{\pi}^{0}(s)\right|^{2}
\end{aligned}
$$

where $\beta_{0}^{3}(s)$ is the threshold kinematic factor and $F_{\pi}^{0}(s)$ the pion form factor. On the $\tau$ side, the physics is contained in the hadronic mass distribution and one can write correspondingly to Eq. (3)

$$
\begin{aligned}
\frac{1}{\Gamma} \frac{d \Gamma}{d s}\left(\tau \longrightarrow \pi^{-} \pi^{0} \nu_{\tau}\right) & = \\
\frac{6 \pi\left|V_{u d}\right|^{2} S_{E W}}{m_{\tau}^{2}} & \frac{B_{e}}{B_{\pi \pi^{0}}} C(s) v_{-}(s) \\
v_{-}(s) & =\frac{\beta_{-}^{3}(s)}{12 \pi}\left|F_{\pi}^{-}(s)\right|^{2}
\end{aligned}
$$

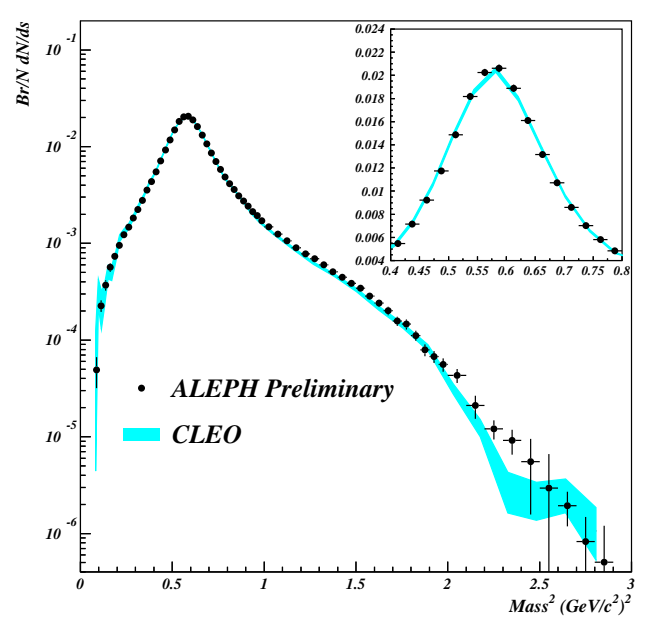

Figure 4. The comparison between ALEPH and CLEO spectral functions in the $\pi \pi^{0}$ mode.

$$
C(s)=\left(1-\frac{s}{m_{\tau}^{2}}\right)\left(1+\frac{2 s}{m_{\tau}^{2}}\right)
$$

SU(2) symmetry (CVC) implies

$v_{-}(s)=v_{0}(s)$

It is important to note that experiments on $\tau$ decays measure the rate inclusive of radiative photons, i.e. for $\tau \rightarrow \nu_{\tau} \pi \pi^{0}(\gamma)$. The measured spectral function is thus $v_{-}^{*}(s)=v_{-}(s) G(s)$, where $G(s)$ is a radiative correction. Also, as $\left|F_{\pi}^{0}(s)\right|^{2}$ should only involve the pion hadronic structure, it must not contain vacuum polarization in the photon propagator.

Three levels of $\mathrm{SU}(2)$ breaking can be identified:

- electroweak radiative corrections to $\tau$ decays are contained in the $S_{\mathrm{EW}}$ factor $[7,8]$ which is dominated by short-distance effects. As such it is expected to be weakly dependent on the specific hadronic final state, as verified by detailed computations in the $\tau \longrightarrow(\pi, K) \nu_{\tau}$ channels [9]. Recently, detailed calculations have been performed for 
the $\pi \pi^{0}$ channel [10] which also confirm the relative smallness of the long-distance contributions. One can write the total correction as

$S_{E W}=\frac{S_{E W}^{\mathrm{had}} S_{E M}^{\mathrm{had}}}{S_{E M}^{\mathrm{lep}}}$

where $S_{E W}^{\text {had }}$ is the leading-log short-distance electroweak factor (which vanishes for leptons) and $S_{E M}^{\text {had,lep }}$ are the nonleading electromagnetic corrections. The latter corrections are calculated in Ref. [8] at the quark level and in Ref. [10] at the hadron level for the $\pi \pi^{0}$ decay mode, and in Ref. $[7,8]$ for leptons. The total correction amounts [13] to $S_{E W}^{\text {inclu }}=1.0198 \pm 0.0006$ for the inclusive hadron decay rate and $S_{E W}^{\pi \pi^{0}}=(1.0232 \pm$ $0.0006) S_{E M}^{\pi \pi^{0}}(s)$ for the $\pi \pi^{0}$ decay mode, where $S_{E M}^{\pi \pi^{0}}(s)$ is an $s$-dependent radiative correction [10].

- pion mass splitting, which is almost completely from electromagnetic origin, directly breaks isospin symmetry in the spectral functions $[11,12]$ since $\beta_{-}(s) \neq \beta_{0}(s)$.

- symmetry is also broken in the pion form factor $[11,10]$. The $\rho$ width is affected by $\pi$ and $\rho$ mass splittings and by explicit electromagnetic decays such as $\pi \gamma, \eta \gamma, l^{+} l^{-}$ and $\pi \pi \gamma$. Isospin violation in the strong amplitude is expected to be negligible because of the small absolute mass difference between $u$ and $d$ quarks.

\subsubsection{Fitting the $2 \pi$ Spectral Function}

The $2 \pi$ spectral function is dominated by the $\rho$ resonance. This provides us with an opportunity to study the relevant description of a wide hadronic resonance. The Gounaris-Sakurai [14] (GS) parametrization satisfies analyticity and unitarity through finite width corrections. The line shape has to be modified to account for the effect of $\rho-\omega$ interference.

The pion form factor is fitted with interfering amplitudes from $\rho, \rho^{\prime}$ and $\rho^{\prime \prime}$ vector mesons with relative strengths $1, \beta$ and $\gamma$. A phase $\phi_{\beta}$ is also considered, since the relative phase of the $\rho$ and $\rho^{\prime}$ amplitude is a priori unknown. In practice we fit the $F_{\pi}^{0}(s)$ form factor from $e^{+} e^{-}$data and the $F_{\pi}^{-}(s)$ form factor from the $\tau$ spectral function duly corrected for $\mathrm{SU}(2)$ breaking, however only in the spectral function phase space and in the $S_{E W}$ factor. In this way, the two form factors can be readily compared, i.e. the masses and widths of the dominating $\rho$ resonance in the two isospin states can be unambiguously determined. On the $e^{+} e^{-}$side, all available data have been used (complete references can be found in Ref. [13], except those of NA7 [15] which are questionable [16]. However, in the $\rho$ mass region the new precise results from CMD-2 [17] are dominating. On the $\tau$ side, the accurate data from ALEPH and CLEO are used.

The systematic uncertainties are included in the fits through appropriate covariance matrices. The $\rho$ mass systematic uncertainty in $\tau$ data is mostly from calibration $(0.7 \mathrm{MeV}$ for ALEPH and $0.9 \mathrm{MeV}$ for CLEO). The corresponding uncertainties on the $\rho$ width are 0.8 and $0.7 \mathrm{MeV}$. Both mass and width determinations are systematically limited in $\tau$ data, however they are uncorrelated between ALEPH and CLEO. Due to the large event statistics, the fits are quite sensitive to the precise line shape and to the interference between the different amplitudes. Of course, $\rho-\omega$ interference is included for $e^{+} e^{-}$data only and the corresponding amplitude $\left(\alpha_{\rho \omega}\right)$ fitted. Fits are performed separately for the $\tau$ and $e^{+} e^{-}$form factors. The upper range of the fit is taken at 3.6 $\mathrm{GeV}^{2}$ for $e^{+} e^{-}$, but at $2.4 \mathrm{GeV}^{2}$ for $\tau$ to avoid the extreme edge of the spectral function where large corrections are applied.

It turns out that the fitted $\rho$ masses and widths (essentially the latter) are quite sensitive to the strength of the $\rho^{\prime}$ and $\rho^{\prime \prime}$ amplitudes, $\beta$ and $\gamma$. So, depending on the type of fit, the derived values exhibit some systematic shifts. The $e^{+} e^{-}$and $\tau$ fits yield significantly different $\rho^{\prime}$ amplitudes and phases: the phase in the $\tau$ fit is consistent with $180^{\circ}$, while it comes out $30^{\circ}$ smaller in the $e^{+} e^{-}$fit. This is merely a reflection of the discrepancy between the measurements of the pion form factor, especially between 0.8 and $1.0 \mathrm{GeV}$. A fit to both data sets is also performed, keep- 


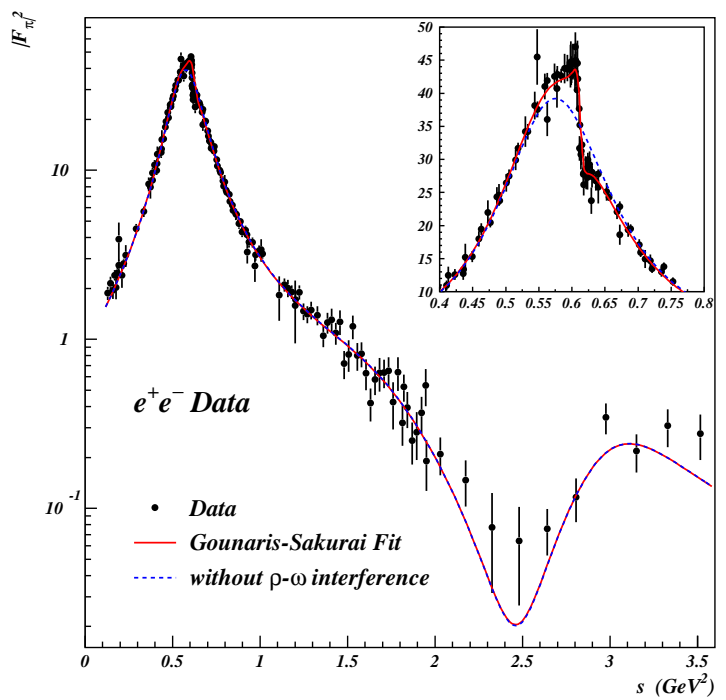

Figure 5. The pion form factor squared $\left|F_{\pi}^{0}(s)\right|^{2}$ from $e^{+} e^{-}$data and the combined fit from Table 1 .

ing common values for the $\rho^{\prime}$ and $\rho^{\prime \prime}$ parameters (second-order with respect to the dominant investigated $\rho$ parameters). Table 1 presents the results of the common fits, the quality of which can be inspected in Figs. 5 and 6 .

The differences found between the masses and widths of the charged and neutral $\rho$ 's are somewhat larger than predicted by Chiral Perturbation Theory $(\chi \mathrm{PT})[18]\left(\left|m_{\rho^{-}}-m_{\rho^{0}}\right|<0.7 \mathrm{MeV}\right)$ and isospin breaking $\left(\Gamma_{\rho^{-}}-\Gamma_{\rho^{0}}=(0.7 \pm 0.3)\right.$ $\mathrm{MeV})$. However, if the mass difference $(2.6 \mathrm{MeV})$ is taken as an experimental fact, then the larger observed width difference $(3.1 \mathrm{MeV})$ could be explained by $\chi \mathrm{PT}(0.7 \mathrm{MeV}$ from isospin breaking $+1.5 \mathrm{MeV}$ from the mass difference). Whether or not these discrepancies are of experimental or theoretical nature is still open [13,19] and needs to be further investigated.

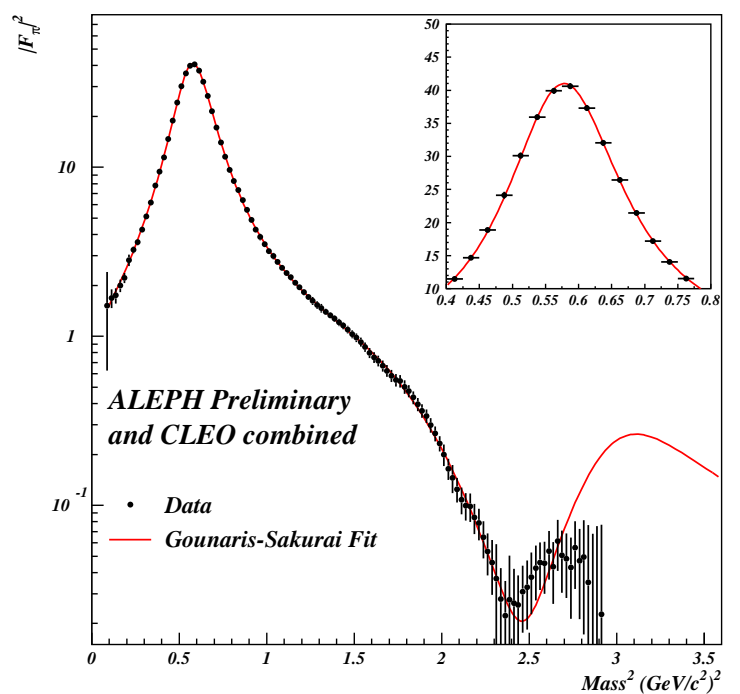

Figure 6 . The pion form factor squared $\left|F_{\pi}^{-}(s)\right|^{2}$ from $\tau$ data and the combined fit from Table 1 .

\subsection{The $4 \pi$ Vector State}

The $4 \pi$ final states have also been studied [3, 20]. Tests of CVC are severely hampered by large deviations between different $e^{+} e^{-}$experiments which disagree beyond their quoted systematic uncertainties. A CLEO analysis studies the resonant structure in the $3 \pi \pi^{0}$ channel which is shown to be dominated by $\omega \pi$ and $a_{1} \pi$ contributions. The $\omega \pi$ spectral function is interpreted by a sum of $\rho$-like amplitudes. The mass of the second state is however found at $(1523 \pm 10) \mathrm{MeV}$, in contrast with the value $(1406 \pm 14) \mathrm{MeV}$ from the fit of the $2 \pi$ spectral function. This point has to be clarified. Following a limit of $8.6 \%$ obtained earlier by ALEPH [21], CLEO sets a new $95 \%$ CL limit of $6.4 \%$ for the relative contribution of second-class currents in the decay $\tau \rightarrow \nu_{\tau} \pi^{-} \omega$ from the hadronic angular decay distribution.

The new ALEPH spectral function in the $3 \pi \pi^{0}$ mode is shown in Fig. 7: it compares well with the CLEO published results [20], except maybe in the threshold region near $1 \mathrm{GeV}$. 
Table 1

\begin{tabular}{|c|c|c|c|}
\hline & $\tau$ & $e^{+} e^{-}$ & combined \\
\hline \hline$m_{\rho^{0}}$ & - & $773.3 \pm 0.6$ & $772.7 \pm 0.5$ \\
$\Gamma_{\rho^{0}}$ & - & $145.2 \pm 1.3$ & $146.4 \pm 0.9$ \\
$m_{\rho^{-}}$ & $775.0 \pm 0.6$ & - & $775.3 \pm 0.6$ \\
$\Gamma_{\rho^{-}}$ & $149.5 \pm 1.1$ & - & $149.5 \pm 0.8$ \\
\hline$\alpha_{\rho \omega}$ & - & $(2.02 \pm 0.10) 10^{-3}$ & $(1.98 \pm 0.10) 10^{-3}$ \\
\hline$\beta$ & $0.195 \pm 0.028$ & $0.123 \pm 0.011$ & $0.172 \pm 0.006$ \\
$\phi_{\beta}$ & $173.0 \pm 7.0$ & $139.4 \pm 6.5$ & $178.2 \pm 4.5$ \\
$m_{\rho^{\prime}}$ & $1440 \pm 34$ & $1337 \pm 35$ & $1415 \pm 15$ \\
$\Gamma_{\rho^{\prime}}$ & $597 \pm 102$ & $569 \pm 81$ & $528 \pm 42$ \\
\hline$\gamma$ & $0.095 \pm 0.029$ & $0.048 \pm 0.008$ & $0.072 \pm 0.006$ \\
$\phi_{\gamma}$ & 0. & 0. & 0. \\
$m_{\rho^{\prime \prime}}$ & 1713 & $1713 \pm 15$ & $1741 \pm 20$ \\
$\Gamma_{\rho^{\prime \prime}}$ & 235 & 235 & 235 \\
\hline \hline
\end{tabular}

Results of fits to the pion form factor squared to $\tau$ and $e^{+} e^{-}$data (ALEPH and CLEO) separately, then combined. The parametrization of the $\rho$ line shape (Gounaris-Sakurai) is described in the text. All mass and width values are in $\mathrm{MeV}$ and the phase $\phi_{\beta}$ in degrees.

The $\tau$ spectral function in the four-pion modes can be used to predict the corresponding isospinrotated $e^{+} e^{-}$cross sections using the $\mathrm{SU}(2)$ symmetric relations

$$
\begin{aligned}
\sigma_{e^{+} e^{-} \rightarrow \pi^{+} \pi^{-} \pi^{+} \pi^{-}}^{I=1} & =2 \cdot \frac{4 \pi \alpha^{2}}{s} v_{\pi^{-} 3 \pi^{0}}, \\
\sigma_{e^{+} e^{-} \rightarrow \pi^{+} \pi^{-} \pi^{0} \pi^{0}}^{I=1} & =\frac{4 \pi \alpha^{2}}{s}\left[v_{2 \pi^{-} \pi^{+} \pi^{0}}-v_{\pi^{-} 3 \pi^{0}}\right]
\end{aligned}
$$

Comparisons can be performed after applying known isospin-breaking corrections $[12,13]$. As shown in Figs. 8 and 9, agreement is observed in the $2 \pi^{+} 2 \pi^{-}$mode, whereas the situation is confused in the $\pi^{+} \pi^{-} 2 \pi^{0}$ mode where a large spread exists between the different $e^{+} e^{-}$results (references can be found in Ref. [13]).

\subsection{Axial-vector States}

The decay $\tau \rightarrow \nu_{\tau} 3 \pi$ is the cleanest place to study axial-vector resonance structure. The spectrum is dominated by the $1^{+} a_{1}$ state, known to decay essentially through $\rho \pi$. A comprehensive analysis of the $\pi^{-} 2 \pi^{0}$ channel has been presented by CLEO. First, a model-independent determination of the hadronic structure functions gave no evidence for non-axial-vector contributions (< $17 \%$ at $90 \%$ CL) [22]. Second, a partial-wave am- plitude analysis was performed [23]: while the dominant $\rho \pi$ mode was of course confirmed, it came as a surprize that an important contribution $(\sim 20 \%)$ from scalars $\left(\sigma, f_{0}(1470), f_{2}(1270)\right)$ was found in the $2 \pi$ system.

The $a_{1} \rightarrow \pi^{-} 2 \pi^{0}$ precisely determined line shape shows the opening of the $K^{*} K$ decay mode in the total $a_{1}$ width. The derived branching ratio, $B\left(a_{1} \rightarrow K^{*} K\right)=(3.3 \pm 0.5) \%$ is in good agreement with ALEPH results on the $K \bar{K} \pi$ modes which were indeed shown (with the help of $e^{+} e^{-}$data and CVC) to be axial-vector $\left(a_{1}\right)$ dominated with $B\left(a_{1} \rightarrow K^{*} K\right)=(2.6 \pm 0.3) \%$ [5]. No conclusive evidence for a higher mass state $\left(a_{1}^{\prime}\right)$ was found in this analysis.

\section{Inclusive Nonstrange Spectral Func- tions}

The $\tau$ nonstrange spectral functions have been measured by ALEPH [3,4] and OPAL [24]. The procedure requires a careful separation of vector (V) and axial-vector (A) states involving the reconstruction of multi- $\pi^{0}$ decays and the proper treatment of final states with a $K \bar{K}$ pair. The $V$ and $A$ spectral functions are given in Fig. 10 . They show a strong resonant behaviour, domi- 


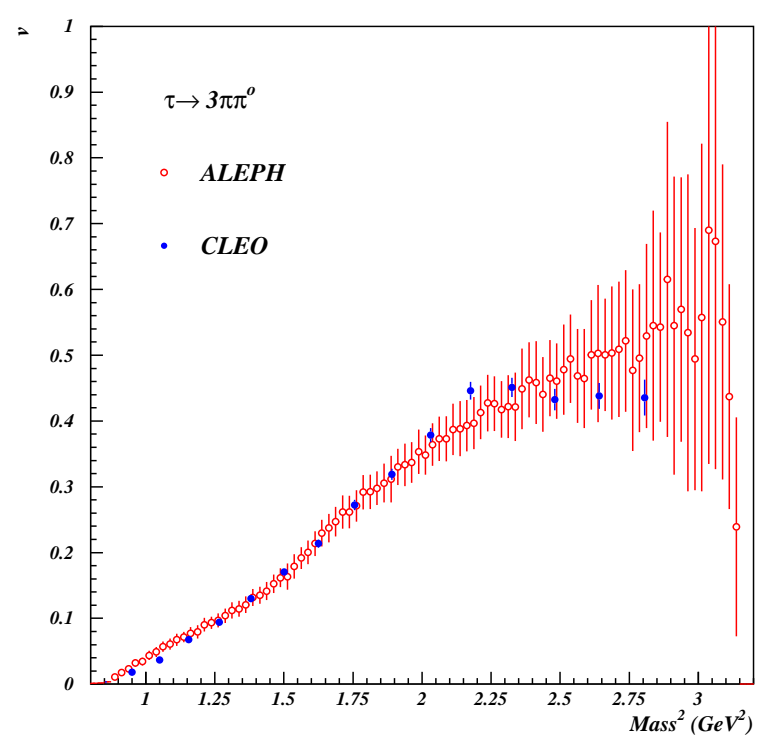

Figure 7. The spectral function for the $3 \pi \pi^{0}$ mode as determined by ALEPH and CLEO.

nated by the lowest $\rho$ and $a_{1}$ states, with a tendancy to converge at large mass toward a value near the parton model expectation. Yet, the vector part stays clearly above while the axial-vector one lies below. Thus, the two spectral functions are clearly not 'asymptotic' at the $\tau$ mass scale.

The new inclusive ALEPH $V$ and $A$ spectral functions are given in Figs. 11 and 12 with a breakdown of the respective contributions.

The $V+A$ spectral function, shown in Fig. 13 has a clear pattern converging toward a value above the parton level as expected in QCD. In fact, it displays a textbook example of global duality, since the resonance-dominated low-mass region shows an oscillatory behaviour around the asymptotic QCD expectation, assumed to be valid in a local sense only for large masses. This feature will be quantitatively discussed in the next section.

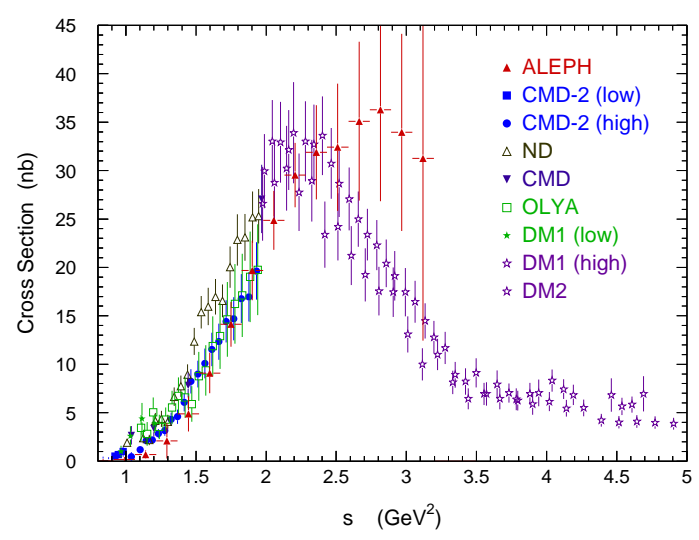

Figure 8. The cross section for $e^{+} e^{-} \rightarrow 2 \pi^{+} 2 \pi^{-}$ from $e^{+} e^{-}$experiments and derived from the $\pi 3 \pi^{0}$ spectral function measured by ALEPH.

\section{QCD Analysis of Nonstrange $\tau$ Decays}

\subsection{Motivation}

The total hadronic $\tau$ width, properly normalized to the known leptonic width,

$R_{\tau}=\frac{\Gamma\left(\tau^{-} \rightarrow \text { hadrons }^{-} \nu_{\tau}\right)}{\Gamma\left(\tau^{-} \rightarrow e^{-} \bar{\nu}_{e} \nu_{\tau}\right)}$

should be well predicted by QCD as it is an inclusive observable. Compared to the similar quantity defined in $e^{+} e^{-}$annihilation, it is even twice inclusive: not only are all produced hadronic states at a given mass summed over, but an integration is performed over all the possible masses from $m_{\pi}$ to $m_{\tau}$.

This favourable situation could be spoiled by the fact that the $Q^{2}$ scale is rather small, so that questions about the validity of a perturbative approach can be raised. At least two levels are to be considered: the convergence of the perturbative expansion and the control of the nonperturbative contributions. Happy circumstances make these latter components indeed very small $[25,26]$. 


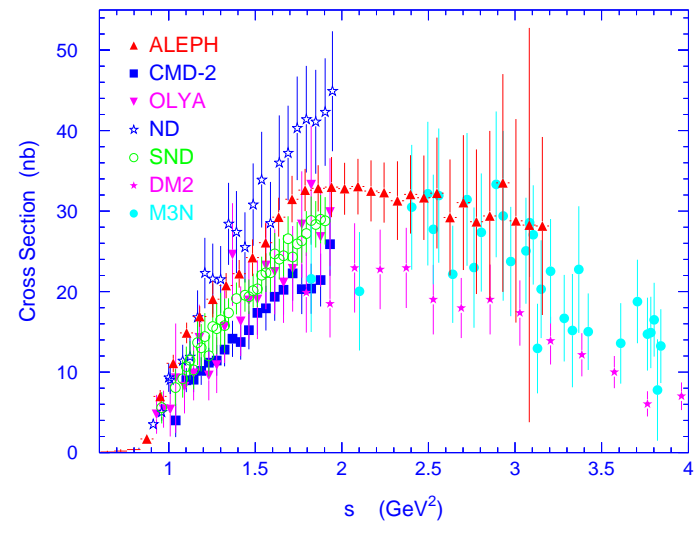

Figure 9. The cross section for $e^{+} e^{-} \rightarrow \pi^{+} \pi^{-} 2 \pi^{0}$ from $e^{+} e^{-}$experiments and derived from the $\pi 3 \pi^{0}$ and $3 \pi \pi^{0}$ spectral functions measured by ALEPH.

\subsection{Theoretical Prediction for $\boldsymbol{R}_{\tau}$}

The imaginary parts of the vector and axial-vector two-point correlation functions $\Pi_{\bar{u} d, V / A}^{(J)}(s)$, with the spin $J$ of the hadronic system, are proportional to the $\tau$ hadronic spectral functions with corresponding quantum numbers. The non-strange ratio $R_{\tau}$ can be written as an integral of these spectral functions over the invariant mass-squared $s$ of the final state hadrons [27]:

$$
\begin{aligned}
& R_{\tau}\left(s_{0}\right)=12 \pi S_{\mathrm{EW}} \int_{0}^{s_{0}} \frac{d s}{s_{0}}\left(1-\frac{s}{s_{0}}\right)^{2} \\
& \times\left[\left(1+2 \frac{s}{s_{0}}\right) \operatorname{Im} \Pi^{(1)}(s+i \epsilon)+\operatorname{Im} \Pi^{(0)}(s+i \epsilon)\right]
\end{aligned}
$$

By Cauchy's theorem the imaginary part of $\Pi^{(J)}$ is proportional to the discontinuity across the positive real axis.

The energy scale $s_{0}=m_{\tau}^{2}$ is large enough so that contributions from nonperturbative effects are small. It is therefore assumed that one can
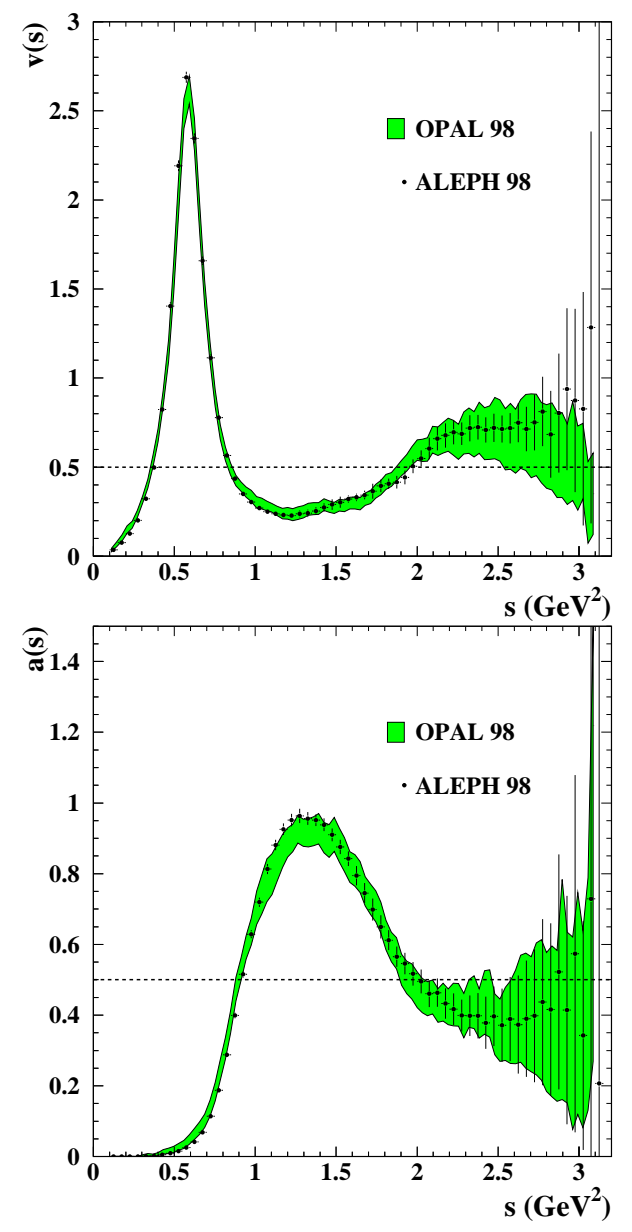

Figure 10. Inclusive nonstrange vector (top) and axial-vector (bottom) spectral functions from ALEPH and OPAL. The dashed line is the expectation from the naive parton model. 


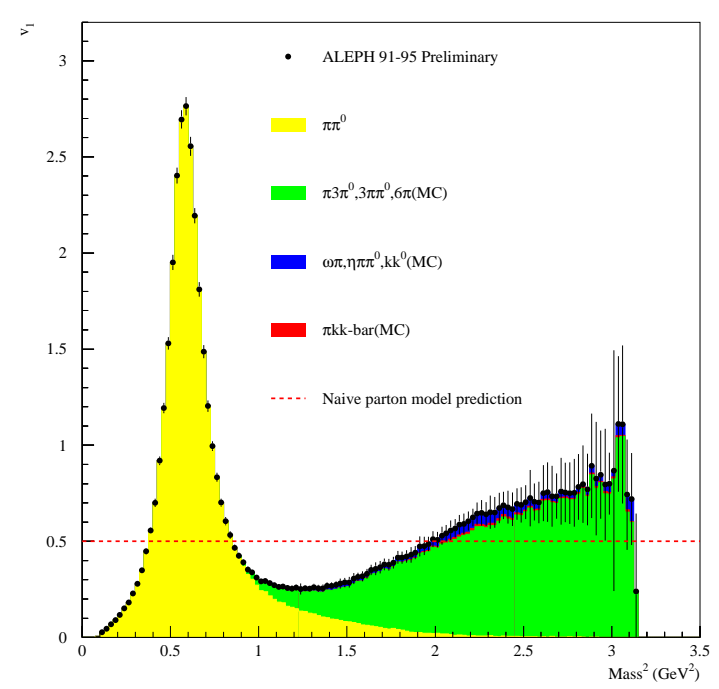

Figure 11. Preliminary ALEPH inclusive vector spectral function with its different contributions. The dashed line is the expectation from the naive parton model.

use the Operator Product Expansion (OPE) to organize perturbative and nonperturbative contributions [28] to $R_{\tau}\left(s_{0}\right)$.

The theoretical prediction of the vector and axial-vector ratio $R_{\tau, V / A}$ can thus be written as:

$$
\begin{aligned}
& R_{\tau, V / A}=\frac{3}{2}\left|V_{u d}\right|^{2} S_{\mathrm{EW}} \\
& \times\left(1+\delta^{(0)}+\delta_{\mathrm{EW}}^{\prime}+\delta_{u d, V / A}^{(2-\mathrm{mass})}+\sum_{D=4,6,8} \delta_{u d, V / A}^{(D)}\right)
\end{aligned}
$$

with the residual non-logarithmic electroweak correction $\delta_{\mathrm{EW}}^{\prime}=0.0010$ [8], neglected in the following, and the dimension $D=2$ contribution $\delta_{u d, V / A}^{(2-\text { mass })}$ from quark masses which is lower than $0.1 \%$ for $u, d$ quarks. The term $\delta^{(0)}$ is the purely perturbative contribution, while the $\delta^{(D)}$ are the OPE terms in powers of $s_{0}^{-D / 2}$ of the following form

$\delta_{u d, V / A}^{(D)} \sim \sum_{\operatorname{dim} \mathcal{O}=D} \frac{\left\langle\mathcal{O}_{u d}\right\rangle_{V / A}}{\left(-s_{0}\right)^{D / 2}}$

where the long-distance nonperturbative effects

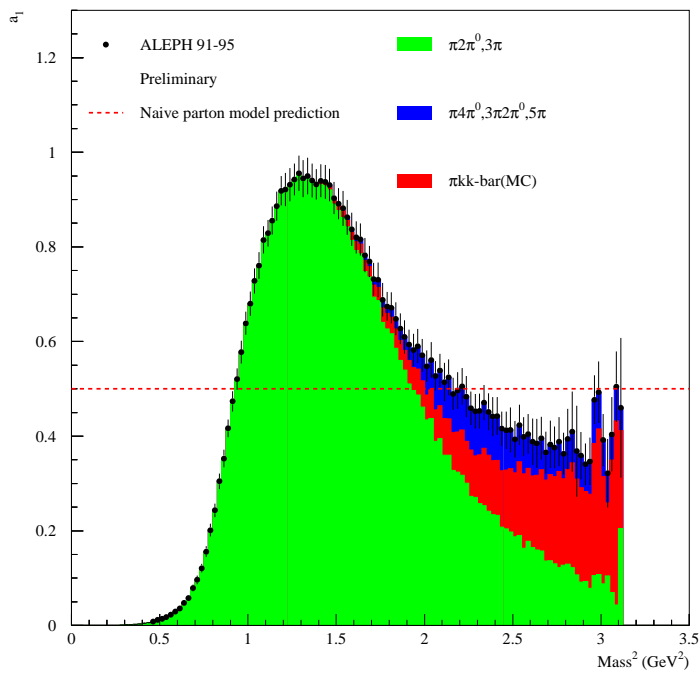

Figure 12. Preliminary ALEPH inclusive axialvector spectral function with its different contributions. The dashed line is the expectation from the naive parton model.

are absorbed into the vacuum expectation elements $\left\langle\mathcal{O}_{u d}\right\rangle$.

The perturbative expansion (FOPT) is known to third order [29]. A resummation of all known higher order logarithmic integrals improves the convergence of the perturbative series (contourimproved method $\mathrm{FOPT}_{\mathrm{CI}}$ ) [30]. As some ambiguity persists, the results are given as an average of the two methods with the difference taken as a systematic uncertainty.

\subsection{Measurements}

The QCD analysis of the $\tau$ hadronic width has not yet been completed with the final ALEPH spectral functions. Results given below correspond to the published analyses with a smaller data set.

The ratio $R_{\tau}$ is obtained from measurements of the leptonic branching ratios:

$R_{\tau}=3.647 \pm 0.014$

using a value $B\left(\tau^{-} \rightarrow e^{-} \bar{\nu}_{e} \nu_{\tau}\right)=(17.794 \pm$ $0.045) \%$ which includes the improvement in ac- 


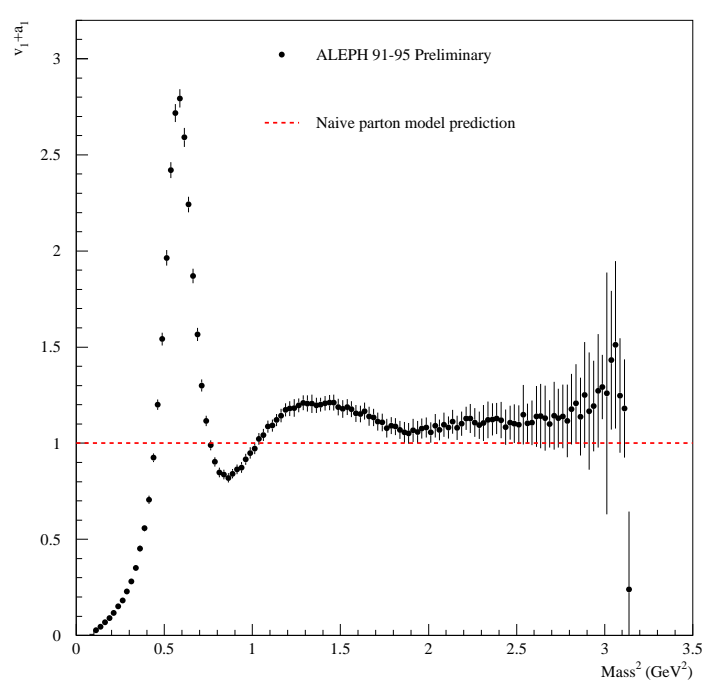

Figure 13. The preliminary inclusive $V+A$ nonstrange spectral function from ALEPH. The dashed line is the expectation from the naive parton model.

curacy provided by the universality assumption of leptonic currents together with the measurements of $B\left(\tau^{-} \rightarrow e^{-} \bar{\nu}_{e} \nu_{\tau}\right), B\left(\tau^{-} \rightarrow \mu^{-} \bar{\nu}_{\mu} \nu_{\tau}\right)$ and the $\tau$ lifetime. The nonstrange part of $R_{\tau}$ is obtained by subtracting out the measured strange contribution (see last section).

Two complete analyses of the $V$ and $A$ parts have been performed by ALEPH [4] and OPAL [24]. Both use the world-average leptonic branching ratios, but their own measured spectral functions. The results on $\alpha_{s}\left(m_{\tau}^{2}\right)$ are therefore strongly correlated and indeed agree when the same theoretical prescriptions are used.

\subsection{Results of the Fits}

The results of the fits are given in Table 2 for the ALEPH analysis. Similar results are obtained by OPAL. It is worth emphasizing that the nonperturbative contributions are found to be very small, as expected. The limited number of observables and the strong correlations between the spectral moments introduce large correlations, especially between the fitted nonperturbative oper-

\begin{tabular}{|l||c|c|}
\hline ALEPH & $\alpha_{s}\left(m_{\tau}^{2}\right)$ & $\delta_{\mathrm{NP}}$ \\
\hline $\mathrm{V}$ & $0.330 \pm 0.014 \pm 0.018$ & $0.020 \pm 0.004$ \\
$\mathrm{~A}$ & $0.339 \pm 0.013 \pm 0.018$ & $-0.027 \pm 0.004$ \\
\hline $\mathrm{V}+\mathrm{A}$ & $0.334 \pm 0.007 \pm 0.021$ & $-0.003 \pm 0.004$ \\
\hline
\end{tabular}

Table 2

Fit results of $\alpha_{s}\left(m_{\tau}^{2}\right)$ and the OPE nonperturbative contributions from vector, axial-vector and $(V+A)$ combined fits using the corresponding ratios $R_{\tau}$ and the spectral moments as input parameters. The second error is given for theoretical uncertainty.

ators.

One notices a remarkable agreement within statistical errors between the $\alpha_{s}\left(m_{\tau}^{2}\right)$ values using vector and axial-vector data. The total nonperturbative power contribution to $R_{\tau, V+A}$ is compatible with zero within an uncertainty of $0.4 \%$, that is much smaller than the error arising from the perturbative term. This cancellation of the nonperturbative terms increases the confidence on the $\alpha_{s}\left(m_{\tau}^{2}\right)$ determination from the inclusive $(V+A)$ observables.

The final result from ALEPH is :

$\alpha_{s}\left(m_{\tau}^{2}\right)=0.334 \pm 0.007_{\exp } \pm 0.021_{\text {th }}$

where the first error accounts for the experimental uncertainty and the second gives the uncertainty of the theoretical prediction of $R_{\tau}$ and the spectral moments as well as the ambiguity of the theoretical approaches employed.

In the OPAL analysis the corresponding results are quoted within three prescriptions for the perturbative expansion, respectively $\mathrm{FOPT}_{\mathrm{CI}}$, FOPT and with renormalon chains, i.e.

$$
\begin{aligned}
\alpha_{s}\left(m_{\tau}^{2}\right) & =0.348 \pm 0.009_{\exp } \pm 0.019_{\mathrm{th}} \\
& =0.324 \pm 0.006_{\exp } \pm 0.013_{\mathrm{th}} \\
& =0.306 \pm 0.005_{\exp } \pm 0.011_{\mathrm{th}}
\end{aligned}
$$

\subsection{Test of the Running of $\alpha_{s}(s)$ at Low Energies}

Using the spectral functions, one can simulate the physics of a hypothetical $\tau$ lepton with a mass $\sqrt{s_{0}}$ smaller than $m_{\tau}$ through equation (7) and hence further investigate QCD phenomena 


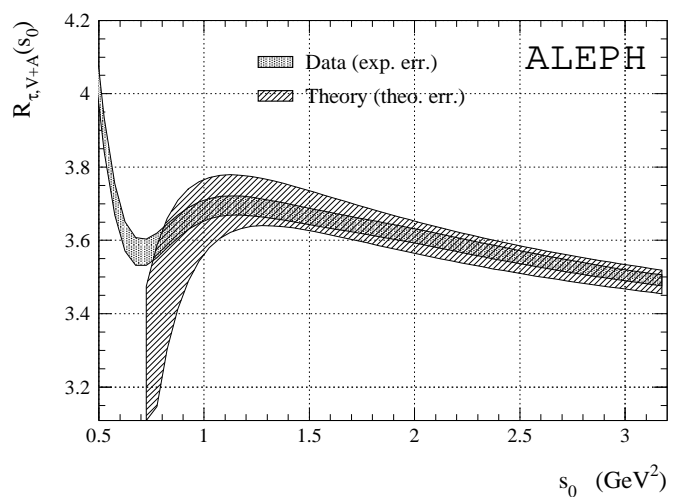

Figure 14. The ratio $R_{\tau, V+A}$ versus the square " $\tau$ mass" $s_{0}$. The curves are plotted as error bands to emphasize their strong point-to-point correlations in $s_{0}$. Also shown is the theoretical prediction using the results of the fit at $s_{0}=m_{\tau}^{2}$.

at low energies. Assuming quark-hadron duality, the evolution of $R_{\tau}\left(s_{0}\right)$ provides a direct test of the running of $\alpha_{s}\left(s_{0}\right)$, governed by the RGE $\beta$ function. On the other hand, it is a test of the validity of the OPE approach in $\tau$ decays.

The functional dependence of $R_{\tau, V+A}\left(s_{0}\right)$ is plotted in Fig. 14 together with the theoretical prediction using the results of Table 2. Below $1 \mathrm{GeV}^{2}$ the error of the theoretical prediction of $R_{\tau, V+A}\left(s_{0}\right)$ starts to blow up due to the increasing uncertainty from the unknown fourth-order perturbative term. Fig. 15 has the same physical content as Fig. 14, but translated into the running of $\alpha_{s}\left(s_{0}\right)$, i.e., the experimental value for $\alpha_{s}\left(s_{0}\right)$ has been individually determined at every $s_{0}$ from the comparison of data and theory. Good agreement is observed with the four-loop RGE evolution using three quark flavours.

The experimental fact that the nonperturbative contributions cancel over the whole range $1.2 \mathrm{GeV}^{2} \leq s_{0} \leq m_{\tau}^{2}$ leads to confidence that the $\alpha_{s}$ determination from the inclusive $(V+A)$ data is robust.

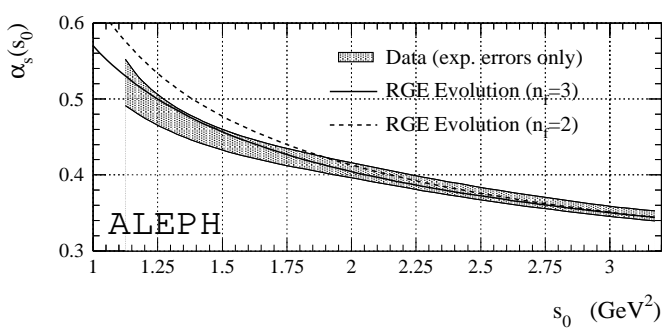

Figure 15. The running of $\alpha_{s}\left(s_{0}\right)$ obtained from the fit of the theoretical prediction to $R_{\tau, V+A}\left(s_{0}\right)$. The shaded band shows the data including experimental errors. The curves give the four-loop RGE evolution for two and three flavours.

\subsection{Discussion on the Determination of $\alpha_{s}\left(m_{\tau}^{2}\right)$}

The evolution of the $\alpha_{s}\left(m_{\tau}^{2}\right)$ measurement from the inclusive $(V+A)$ observables based on the Runge-Kutta integration of the differential equation of the renormalization group to $\mathrm{N}^{3} \mathrm{LO}[31,33]$ yields for the ALEPH analysis

$\alpha_{s}\left(M_{\mathrm{Z}}^{2}\right)=0.1202 \pm 0.0008_{\mathrm{exp}} \pm 0.0024_{\mathrm{th}} \pm 0.0010_{\mathrm{evol}}$

where the last error stands for possible ambiguities in the evolution due to uncertainties in the matching scales of the quark thresholds [33].

The result (15) can be compared to the precise determination from the measurement of the $Z$ width, as obtained in the global electroweak fit. The variable $R_{Z}$ has similar advantages to $R_{\tau}$, but it differs concerning the convergence of the perturbative expansion because of the much larger scale. It turns out that this determination is dominated by experimental errors with very small theoretical uncertainties, i.e. the reverse of the situation encountered in $\tau$ decays. The most recent value [34] yields $\alpha_{s}\left(M_{\mathrm{Z}}^{2}\right)=0.1183 \pm 0.0027$, in excellent agreement with (15). Fig. 16 illustrates well the agreement between the evolution of $\alpha_{s}\left(m_{\tau}^{2}\right)$ predicted by QCD and $\alpha_{s}\left(M_{\mathrm{Z}}^{2}\right)$. 


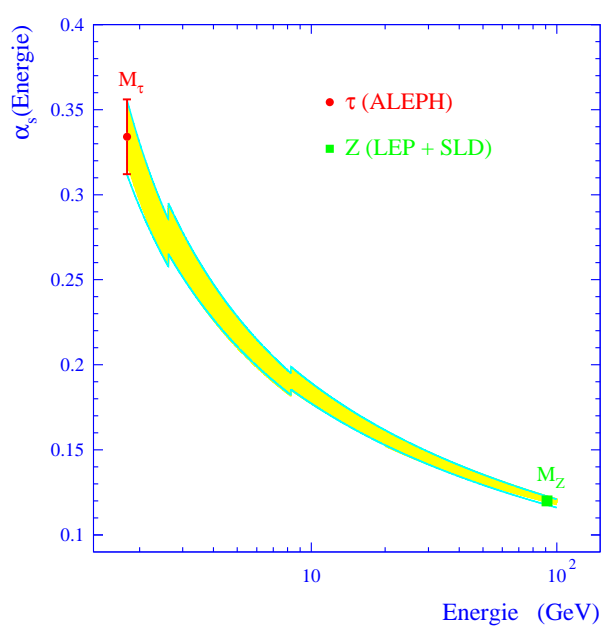

Figure 16. Evolution of the strong coupling (measured at $m_{\tau}^{2}$ ) to $M_{Z}^{2}$ predicted by QCD compared to the direct measurement. The evolution is carried out at 4 loops, while the flavour matching is accomplished at 3 loops at $2 m_{c}$ and $2 m_{b}$ thresholds.

\section{Strange Spectral Function and Strange Quark Mass}

The spectral function for strange final states has been determined by ALEPH [5]. Fig. 17 shows that it is dominated by the vector $K^{*}(890)$ and higher mass (mostly axial-vector) resonances, leading into a poorly defined continuum region, however in agreement with the quark model.

The total rate for strange final states, using the complete ALEPH analyses supplemented by results from other experiments [35] is determined to be $B\left(\tau \rightarrow \nu_{\tau}\right.$ hadrons $\left._{S=-1}\right)=(29.3 \pm 1.0) 10^{-3}$, leading to

$R_{\tau, S}=0.163 \pm 0.006$.

Spectral moments are again useful tools to unravel the different components of the inclusive rate. Since we are mostly interested in the specific contributions from the $\bar{u} s$ strange final state, it is useful to form the difference

$\Delta_{\tau}^{k l} \equiv \frac{1}{\left|V_{u d}\right|^{2}} R_{\tau, S=0}^{k l}-\frac{1}{\left|V_{u s}\right|^{2}} R_{\tau, S=-1}^{k l}$

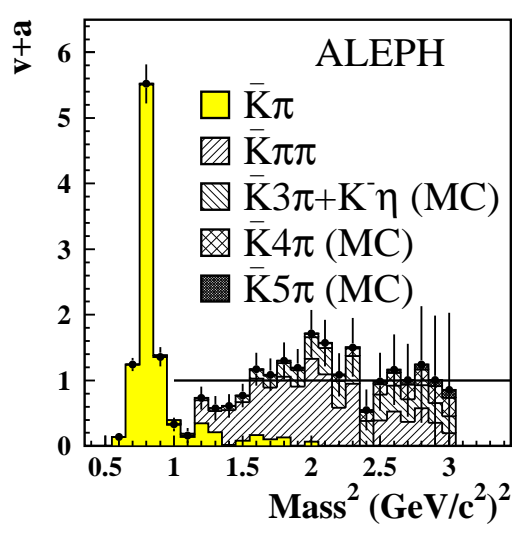

Figure 17. The ALEPH strange spectral function with various contributions indicated. Highermass contributions from final states with larger multiplicities lead to very small branching fractions and are estimated.

where the flavour-independent perturbative part and gluon condensate cancel. Fig. 18 shows the interesting behaviour of $\Delta_{\tau}^{00}$ expressed differentially as a function of $s$.

The leading QCD contribution to $\Delta_{\tau}^{k l}$ is a term proportional to the square of the strange quark mass at the $\tau$ energy scale. Special attention must be devoted to the perturbative expansion of this term which has the early behaviour of an asymptotic series. We quote here the recent result from the analysis of Ref. [36], yielding

$m_{s}\left(m_{\tau}^{2}\right)=\left(120 \pm 11_{\exp } \pm 8_{V_{u s}} \pm 19_{t h}\right) \mathrm{MeV}(18)$

where the dominant uncertainty is from theory, mostly because of the poor convergence behaviour. Stability checks have been performed and their effect has been conservatively taken into account in the estimate of the theoretical uncertainty. 


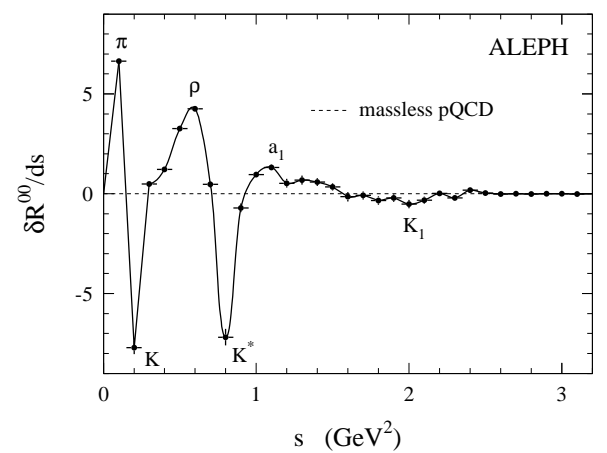

Figure 18. Differential rate for $\Delta_{\tau}^{00}$, difference between properly normalized nonstrange and strange spectral functions (see text for details). The contribution from massless perturbative QCD vanishes. To guide the eye, the solid line interpolates between bins of constant $0.1 \mathrm{GeV}^{2}$ width.

\section{Conclusions}

The decays $\tau \rightarrow \nu_{\tau}+$ hadrons constitute a clean and powerful way to study hadronic physics up to $\sqrt{s} \sim 1.8 \mathrm{GeV}$. Beautiful resonance analyses have already been done, providing new insight into hadron dynamics. Probably the major surprise has been the fact that inclusive hadron production is well described by perturbative QCD with very small nonperturbative components at the $\tau$ mass. Despite the fact that this low-energy region is dominated by resonance physics, methods based on global quark-hadron duality work indeed very well.

The new ALEPH preliminary results using the full LEP1 sample have been presented. Satisfactory agreement with CLEO is observed in the $\pi \pi^{0}$ and $3 \pi \pi^{0}$ decay modes. The $\tau$ spectral functions have now reached a precision level where detailed investigations are possible, particularly in the most interesting $\pi \pi^{0}$ channel. The breaking of $\mathrm{SU}(2)$ symmetry can be directly determined through the comparison between $e^{+} e^{-}$ and $\tau$ spectral functions. The difference between the two spectral functions does not agree with the computed effect of isospin symmetry breaking and requires further experimentation. The determination of the widths of the charged and the neutral $\rho$ is quite sensitive to the fit conditions, even though the data cover a very large mass range.

The measurement of the vector and axialvector spectral functions has provided the way for quantitative QCD analyses. These spectral functions are very well described in a global way by $O\left(\alpha_{s}^{3}\right)$ perturbative QCD with small nonperturbative components. Precise determinations of $\alpha_{s}$ agree for both spectral functions and they also agree with all the other determinations from the $\mathrm{Z}$ width, the rate of $\mathrm{Z}$ to jets and deep inelastic lepton scattering. Indeed from $\tau$ decays

$\alpha_{s}\left(M_{\mathrm{Z}}^{2}\right)_{\tau}=0.1202 \pm 0.0027$

in excellent agreement with the average from all other determinations [37]

$\alpha_{s}\left(M_{\mathrm{Z}}^{2}\right)_{n o n-\tau}=0.1187 \pm 0.0020$

The strange spectral function yields a very competitive value for the strange quark mass which can be evolved to the usual comparison scale

$m_{s}(2 \mathrm{GeV})=\left(116_{-25}^{+20}\right) \mathrm{MeV}$

The use of the vector $\tau$ spectral function and the QCD-based approach as tested in $\tau$ decays improve the calculations of hadronic vacuum polarization considerably. Significant results have been obtained for the running of $\alpha$ to the $\mathrm{Z}$ mass and the muon anomalous magnetic moment. Both of these quantities must be known with high precision as they have the potential to give access to new physics.

\section{Acknowledgments}

I would like to thank my colleagues Shaomin Chen, Andreas Höcker, Changzheng Yuan and Zhiqing Zhang for their many contributions to this work. Fruitful discussions with V. Cirigliano, G. Ecker, S.I. Eidelman, W. Marciano, H. Neufeld, A. Pich and A. Weinstein are acknowledged. Congratulations to Abe Seiden and his staff for organizing an exciting Tau2002 Workshop. 


\section{REFERENCES}

1. Review of Particle Properties, Phys. Rev. D66 (2002) 010001

2. M. Davier and C. Yuan, these proceedings, hep-ex/0211057 (November 2002)

3. R. Barate et al. (ALEPH Collaboration), Z.Phys. C76 (1997) 15

4. ALEPH Collaboration, Eur.Phys.J. C4 (1998) 409

5. ALEPH Collaboration, Eur.Phys.J. C11 (1999) 599

6. S. Anderson et al. (CLEO Collaboration), Phys.Rev. D61(2000) 112002

7. W. Marciano and A. Sirlin, Phys.Rev.Lett. 61 (1988) 1815

8. E. Braaten and C.S. Li, Phys.Rev. 42 (1990) 3888

9. R. Decker and M. Finkenmeier, Nucl.Phys. 438 (1995) 17

10. V. Cirigliano, G. Ecker and H. Neufeld, Phys. Lett. B513 (2001) 361; V. Cirigliano, G. Ecker and H. Neufeld, hep-ph/0207310 (July 2002)

11. R. Alemany, M. Davier and A. Höcker, Eur.Phys.J. C2 (1998) 123

12. H. Czyż and J.H. Kühn, Eur.Phys.J. C18 (2001) 497

13. M. Davier, S.I. Eidelman, A. Höcker and Z. Zhang, hep-ph/0208177 (August 2002)

14. G.J. Gounaris and J.J. Sakurai, Phys. Rev. Lett. 21 (1968) 244

15. S.R. Amendolia et al. (NA7 Collaboration), Phys. Lett. B138 (1984) 454

16. A correction affecting the $s$ scale ought to be applied (L. Rolandi, R. Tenchini, private communications)

17. R.R. Akhmetshin et al. (CMD-2 Collaboration), Phys.Lett. B527 (2002) 161.

18. J. Bijnens and P. Gosdzinsky, Phys. Lett. B388 (1996) 203

19. A. Höcker, these proceedings

20. K.W. Edwards et al. (CLEO Collaboration), Phys.Rev. D61 (2000) 072003

21. D. Buskulic et al. (ALEPH Collaboration), Z. Phys. C74 (1997) 263

22. T.E. Browder et al. (CLEO Collaboration), Phys.Rev. D61(2000) 052004
23. D.M. Asner et al. (CLEO Collaboration), Phys.Rev. D61(2000) 012002

24. OPAL Collaboration, Eur.Phys.J. C7 (1999) 571

25. E. Braaten, Phys.Rev.Lett. 60 (1988) 1606

26. S. Narison and A. Pich, Phys.Lett. B211(1988) 183

27. E. Braaten, S. Narison and A. Pich, Nucl.Phys. B373 (1992) 581

28. M.A. Shifman, A.L. Vainshtein and V.I. Zakharov, Nucl.Phys. B147 (1979) 385; ibid B147 (1979) 448; ibid B147 (1979) 519

29. L.R. Surguladze and M.A. Samuel, Phys. Rev. Lett. 66 (1991) 560; S.G. Gorishny, A.L. Kataev and S.A. Larin, Phys. Lett. B259 (1991) 144

30. F. Le Diberder and A. Pich, Phys. Lett. B286 (1992) 147

31. S.A. Larin, T. van Ritbergen and J.A.M. Vermaseren, Phys. Lett. B400 (1997) 379 K.G. Chetyrkin, B.A. Kniehl and M. Steinhauser, Nucl.Phys. B510 (1998) 61 W. Bernreuther, W. Wetzel, Nucl.Phys. B197 (1982) 228 W. Wetzel, Nucl.Phys. B196 (1982) 259 W. Bernreuther, PITHA-94-31 (1994).

32. K.G. Chetyrkin, B.A. Kniehl and M. Steinhauser, Phys. Rev. Lett. 79 (1997) 2184

33. G. Rodrigo, A. Pich, A. Santamaria, Phys.Lett. B424 (1998) 367

34. LEPEWWG/2002-02, hep-ex/0212036 (December 2002)

35. M. Davier, S. Chen, A. Höcker, J. Prades and A. Pich, Nucl.Phys. (Proc. Suppl.) B98 (2001) 319

36. S. Chen, M. Davier, E. Gámiz, A. Höcker, J. Prades and A. Pich, Eur.Phys.J. C22 (2002) 31

37. M. Davier, '98 Rencontres de Moriond on Electroweak Interactions, Ed. J. Trân Thanh Vân, Frontières, Paris (1998) 